\title{
Hidden Components in Aqueous 'Gold-144' Fractionated by PAGE: High Resolution Orbitrap ESI-MS Identifies the Gold-102 and Higher All-Aromatic Au-pMBA Cluster Compounds
}

\author{
Marcos M. Alvarez ${ }^{1,{ }^{*}}$, Jenny Chen ${ }^{2}$, Germán Plascencia-Villa ${ }^{1}$, David M. Black ${ }^{1}$, Wendell P. \\ Griffith $^{3}$, Ignacio L. Garzon ${ }^{1,4}$, Miguel José Yacamán ${ }^{1}$, Borries Demeler ${ }^{5}$, Robert L. \\ Whetten ${ }^{1, *}$ \\ 1Department of Physics \& Astronomy, University of Texas, San Antonio, TX, 78249, USA. \\ ${ }^{2}$ ThermoFisher Scientific 355 River Oaks Pkwy, San Jose, CA 95134, USA. \\ ${ }^{3}$ RCMI Protein Biomarkers Core. University of Texas, San Antonio, Texas, 78249, USA. \\ ${ }^{4}$ Instituto de Física, Universidad Nacional Autónoma de México, Apartado Postal 20-364, 01000 \\ México, D. F, México \\ ${ }^{5}$ Department of Biochemistry. University of Texas Health Science Center, San Antonio, Texas, \\ 78229.
}

\begin{abstract}
Experimental and theoretical evidence reveals the resilience and stability of the larger aqueous gold clusters protected with $p$-mercaptobenzoic acid ligands ( $p \mathrm{MBA}$ ) of composition $\mathrm{Au}_{\mathrm{n}}(p \mathrm{MBA})_{\mathrm{p}}$ or $(\mathrm{n}, \mathrm{p})$. The $\mathrm{Au}_{144}(p \mathrm{MBA})_{60},(144,60)$, or Gold-144 aqueous gold cluster is considered special because of its high symmetry, abundance, and icosahedral structure as well as its many potential uses in material and biological sciences. Yet to this date, direct confirmation of its precise composition and total structure remains elusive. Results described here from characterization via high-resolution electrospray ionization mass spectrometry on an Orbitrap instrument show $\mathrm{Au}_{102}(p \mathrm{MBA})_{44}$ at isotopic resolution. Further, what usually appears as a single band for $(144,60)$ in electrophoresis (PAGE) is shown to also contain $(130,50)$, recently determined to have a truncated decahedral structure, and $(137,56)$ in addition to the dominant $(144,60)$ compound of chiral icosahedral structure. This finding is significant in that it reveals the existence of structures never before observed in all-aromatic water soluble species while pointing out the path toward elucidation of the thermodynamic control of protected gold nanocrystal formation.
\end{abstract}

\section{Graphical Abstract}

“Correspondence: Marcos.Alvarez@utsa.edu, Robert.Whetten@utsa.edu, Tel: +1 210-458-5451.

Author Contributions The manuscript was written via contributions of all authors. All authors have given approval to the final version of the manuscript.

Notes: The authors declare no competing financial interests.

Supporting Information. Detailed methods, mass spectra and assignments (PDF) 


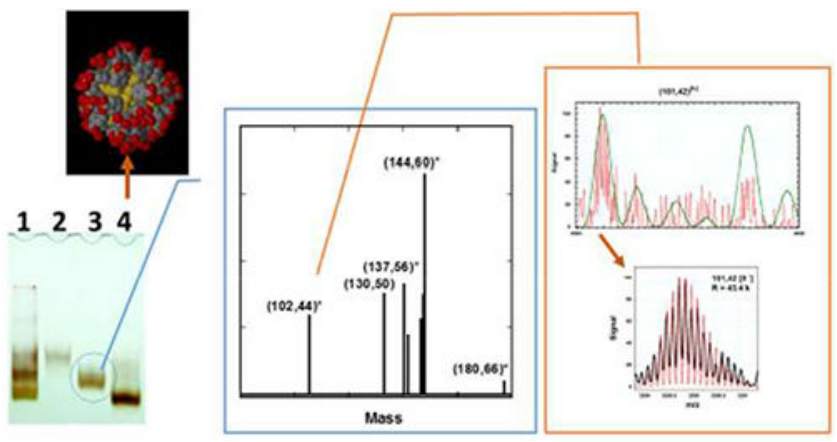

High resolution $(\mathrm{m} / \Delta \mathrm{m}=44 \mathrm{k})$ ESIMS in an Orbitrap analyzer indeed comprises the title $\mathrm{Au}_{144}$ compound but also substantial amounts of $\mathrm{Au}_{137}$ and $\mathrm{Au}_{130}$ protected with the water-soluble allaromatic p-mercaptobenzoic acid ligand, analogous to nonaqueous species.

\section{INTRODUCTION}

From Faraday's colloidal gold to today's gold clusters, ${ }^{1-3}$ one major lesson learned is that the bulk properties of a metal emerge early on at a relatively small cluster size. ${ }^{4-5} \mathrm{~A}$ well defined localized surface plasmon resonance band, for instance, is already established as size independent when the size of the bare metallic core consists of a few dozen atoms. ${ }^{3,5,6-9}$ Evidence for this "golden rule" is apparent in all types of metallic systems but is exceptionally convincing for nonaqueous gold particles protected with organic ligands. ${ }^{10,11}$ In part, this success is due to the amenability of these nonaqueous species to be examined by electrospray ionization mass spectrometry (ESI-MS) and the concomitant plethora of compositional and structural information that can be thereby obtained. ${ }^{5,11-14}$ It is now possible to state with atomic precision the composition (n,p) of a cluster comprised of " $n$ " metal atoms and "p" ligands. Regarding the $(144,60)$ cluster studied here, Dass pointed out that ...

"It was Michael FARADAY (1857) who first explored the small-size limit of the optical properties of metallic gold (and other metals), and who marveled at the extraordinary stability of his thiocarbonate protected gold sols (prepared by combining $\mathrm{P}_{4} / \mathrm{CS}_{2}$ and $\mathrm{NaAuCl}_{4} / \mathrm{H}_{2} \mathrm{O}$ solutions), asking whether these "be considered ... as molecules of gold." "

Larger aqueous clusters $(n>100)$, despite their potential importance in the biomedical sciences, ${ }^{15,16}$ have proved difficult to characterize by mass spectrometry. ${ }^{17-19}$ Added to the difficulty of volatilizing intact these massive species is the formidable assignment challenge on account of byproducts and their propensity to form multiple adducts and charge states. Unlike proteins that had posed similar problems, ${ }^{20-22}$ little progress has been reported in overcoming the above challenges in the characterization of aqueous clusters in the interesting core size range $(1-3 \mathrm{~nm})$ that exhibit both molecular and bulk like properties.

The broader physic-chemical significance of these challenging determinations of compositions and relative abundances can be appreciated via the following brief chronology, dating back nearly a quarter-century: 
In 1992, Wang \& coworkers reported thermodynamic control of nanocrystal formation in the high temperature $C d-S$-SPh system, and thus obtained the ordered $C_{32} S_{14}(S P h)_{36}$ compound (and structure) in high yield. ${ }^{23,24}$ This discovery challenged the prevailing assumption of kinetic control ('arrested precipitation'), and stimulated effort to provide a statistical thermodynamic framework for surface-chemical control of nanocrystal formation.

Toward that end, Gelbart \& coworkers responded to this question — can there exist "nanocrystal micro-emulsions", analogous to the familiar fluid-surfactant-fluid nano-phases? - by demonstrating theoretically that the narrow size-distribution (continuum) of nanodroplets in the latter (fluid) case may become punctuated further in the case of nano crystals, by virtue of the structural 'magic numbers', i.e. thermodynamically preferred morphologies (faceting) and adsorbate (ligand) binding relating to solid surface physical chemistry. ${ }^{25}$

To make this analogy explicit, for the case of large (aq)-Au-pMBA clusters, the metallic $(A u)$ core corresponds to the hydrophobic (oil) interior of the micelle, the $p$ MBA represents the amphiphile (the aurophilic-sulfur inward; the hydrophilic-carboxylate outward), and the aqueous phase is common to both solid state and fluid state microemulsions.

Gelbart \& coworkers ${ }^{25-26}$ considered simplified models incorporating the lattice and faceting of the nanocrystal core, the curvature effect (steric cone-angle) of the surfactant (adsorbate, ligand) tail groups $(R)$, and the chemical potential(s) of the components in solution, to predict equilibrium distributions, and conditions of microemulsion-failure, etc., in the three-component phase diagram.

In this way they obtained an alternative conceptual framework for interpreting the thermodynamic control of ligand-mediated nanocrystal synthesis, or what nowadays is termed by Jin et al. the "size-focusing" methodology. ${ }^{27}$ The self-assembled monolayers, of thiolates-on-gold surfaces, would appear ideal for this approach, given the wealth of fundamental surface physical-chemical studies on the planar phases.

In that same year (1994), Brust et al. reported a high-yield method for obtaining nonaqueous thiolate-protected gold clusters, of core-diameter in the interesting $\sim 2 \pm 1 \mathrm{~nm}$ range. ${ }^{28}$ The Atlanta group then combined separations with rudimentary (LDI)-MS to demonstrate a discrete size-distribution, and used X-ray diffraction to identify high-crystallinity metallic cores. ${ }^{29}$ The Chapel Hill group demonstrated abundantly that the surface chemistry of the thiolate ligands is analogous to that of the planar SAMS.${ }^{30}$ Finally, Gelbart, Heath and coworkers, ${ }^{26}$ made an early attempt to explain the size control via a rudimentary nanocrystal-microemulsions (thermodynamic) model. By 1999, finer control of the conditions for self-assembly would result in very high yields, e.g. $>70 \%$ of the $\sim 30-k D a$ fraction, tentatively identified as $(145,60)$, by Schaaff et al. ${ }^{31}$

However, such systems were neither aqueous nor aromatic. First, aqueous (Au-SG) gold glutathionate clusters were obtained in high yield by Schaaff et al., ${ }^{8}$ separated and quantified by PAGE, and the smallest were analyzed by MALDI-MS and ESI MS, wherein an abundant 10.4- $\mathrm{kDa}$ fraction was assigned the composition $(28,16)$, later revised to $(25,18)$ by Negishi et al., who also obtained MS results for several other fractions, e.g. $(38,24) .{ }^{32}$ Second, all aromatic gold thiolate clusters, i.e. those in which the alpha-carbon is part of a phenyl- 
group, were obtained by Price et al., ${ }^{33}$ primarily as the $29-\mathrm{kDa}$ and $22-\mathrm{kDa}$ species, yet the smaller clusters obtained via oxidative 'etching' of these showed properties quite distinct from the aliphatic counterparts of the same mass range as shown in the early report from Ackerson, Jadzinsky \& Kornberg. ${ }^{34}$

The great breakthrough in aqueous aromatic gold-thiolate clusters came in the 2007 report of the total structure determination of the $\mathrm{Au}_{102}(p \mathrm{MBA})_{44}$ cluster $^{35}$. The authors emphasized that this was not necessarily the main component of the aqueous mixture; a more dominant fraction associated with the (nominal) composition " $\mathrm{Au}_{145}(p \mathrm{MBA})_{60}$ "usually accompanied the title compound. (Both these substances proved refractory to high-resolution ESI-MS analysis.) Later work by Levi-Kalisman et al. ${ }^{36}$ found the narrowly delimited conditions required to obtain $\mathrm{Au}_{102}$ in higher yield. The aqueous clusters of intermediate core size (1 $\mathrm{nm}-3 \mathrm{~nm}$ ), however, remained largely unexplored via mass spectrometry.

We recently reported on the MS characterization of two highly purified fractions of composition $\mathrm{Au}_{102}(p \mathrm{MBA})_{44}-(102,44)$ at $28,832 \mathrm{Da}$, and $\mathrm{Au}_{144}(p \mathrm{MBA})_{60}-(144,60)$ at $37,556 \mathrm{Da} .{ }^{17}$ Here we report progress on the successful characterization of these fractions on an Exactive-Plus-EMR Orbitrap analyzer ${ }^{22}$ at higher resolution $[\mathrm{m} / \Delta \mathrm{m}=44.6 \mathrm{k}$ for $(102,44)$ and $17.5 \mathrm{k}$ for $(144,60)]$. The new findings reiterate the high purity of the $(102,44)$ fraction, now confirmed at isotopic resolution, but also reveal the presence of $(102,44)$, $(130,50),(137,56)$, and $(180,66)$ in the dominant $(144,60)$ fraction. The implementation of high resolution mass spectrometry to massive aqueous species demonstrated here, unveils a wide range of information unprecedented for aqueous clusters including compositional and structural stability information from fragmentation patterns, identification of adducts, and the elucidation of novel cluster species.

\section{EXPERIMENTAL SECTION}

The preparation of larger aqueous Au- $p$ MBA clusters, their purification via gel electrophoresis, and characterization by various methods has been described in detail elsewhere. ${ }^{17}$ To summarize, they were prepared from reduction of gold salts with sodium borohydride in the presence of $p$-MBA as protecting agent. Raw concentrated product was separated into three fractions using native polyacrylamide gel electrophoresis (PAGE, 1012\%), and characterized via Aberration-corrected Atomic Resolution Scanning Transmission Electron Microscopy, dynamic light scattering, absorbance spectroscopy, and zeta-potential. The raw mixture was analyzed via multi-wavelength analytical ultracentrifugation, and the lighter two fractions were analyzed via electrospray ionization mass spectrometry (ESI-MS) on a Bruker micrOTOF instrument. The samples described here are essentially identical (same batches) as those characterized by Plascencia et al. ${ }^{17}$

For the present work, the two lighter fractions were further analyzed on an Exactive Plus EMR Orbitrap spectrometer at the ThermoFisher's facility in San Jose, CA. The spectrometer was operated in negative mode and calibrated using cesium iodide clusters (up to $\mathrm{m} / \mathrm{z}=11,300 \mathrm{Da}$ ). Aqueous solutions were infused at $5 \mu \mathrm{L} / \mathrm{min}$ and a concentration of $\sim$ $0.5 \mu \mathrm{g} / \mu \mathrm{L}$. Details on the relevant instrumental parameters are provided in the supplementary 
information section. Nonaqueous analogous of the species described here were also analyzed under similar conditions, to achieve high resolution at high mass.

\section{RESULTS AND INTERPRETATION}

Mass analysis of the purified aqueous fractions in the Orbitrap instrument resulted in stable signals for both fractions at 140,000 and 17,000 resolution (at $200 \mathrm{Da}$ ) with and without collisional induced dissociation (CID). As with ESI spectra of proteins which exhibit multiple charged states, ${ }^{22}$ the seemingly undecipherable spectra [Figure 1] can be "deconvoluted" into a single dominant peak for $(102,44)$ [Figure 2] and a handful of peaks for $(144,60)$ [Figure 3] using Thermo Protein Deconvolution 4.0 software from ThermoFisher Scientific Inc.

\section{The $(102,44)$ Fraction.}

The seminal $(102,44)$ cluster has been shown to consist of a 79-atom gold core enclosed by nineteen $(1,2)$ monomeric units and two $(2,3)$ polymeric units. ${ }^{35}$ Indeed, ESI-MS spectra of this species is dominated by the $(101,42)$ ions that is attributed to the loss of one monomeric unit, per the following process, ${ }^{17}$

$$
(102,44)^{[(\mathbf{z}+1)-]} \rightarrow(101,42)^{[\mathbf{z}-]}+(\mathbf{1}, 2)^{[-]}
$$

The intact $(102,44)$ parent cluster is not observed in the present experimental conditions. At higher resolution $(44.5 \mathrm{k})$, the various charge-states of the fragment signal from $(101,42)$ can be shown to arise from the loss of a proton (1.0 Da) unit per the following scheme:

$$
\mathbf{M}^{[\mathbf{z}-]} \rightarrow \mathbf{M}^{[(\mathbf{z}+\mathbf{1})-]}+\mathbf{H}^{+}
$$

The charging process results in a shift to lighter mass in the resultant ion signal as the proton is ejected, as summarized in Figure 4. Also evident is the existence of satellite peaks with 22-Da spacing that can be assigned to multiple $\mathrm{Na}^{+}-\mathrm{H}^{+}$cation exchanges occurring at the carboxylate end groups of the protecting $p \mathrm{MBA}$ ligands [Figure 2]. Isotopically resolved mass spectrometric evidence confirming the existence of these $\mathrm{Na}$ adducts is presented in the supplementary information section $\left(\mathrm{Na}^{+}\right.$ions were presumably introduced during the synthesis of nanoclusters from the $\mathrm{NaBH}_{4}$ reductant). Further analysis of the fine structure at the leading edge of the peak also reveals the presence of potassium (of unknown origin), water, and methanol (when used as an ESI co-solvent). Attempts to dissociate the ions via Collision Induced Dissociation yielded complex spectra at higher charge states, attributed to multiple $(1,1),(1,0)$, and $(0,1)$ fragments, in addition to the preexisting adducts.

The presence of these adducts is associated with the difficulty of characterizing the water soluble fractions as the ionic sodium-carboxylate bonds are difficult to break in the ESI process and lead to overly complex spectra when examined at lower resolution. ${ }^{17}$ This finding also hints at a proper course of action in preparations of the aqueous species, i.e. substitute alkali cations in the synthesis with $\left[\mathrm{R}_{3} \mathrm{NH}\right]^{+}$cations which in the ESI-process 
would easily generate charge clusters by evaporation of volatile $\mathrm{NR}_{3}$ groups and retention of $\mathrm{H}^{+},\left(-\mathrm{CO}_{2} \mathrm{H}\right)$. The presence of the proton is essential.

\section{The $(144,60)$ Fraction}

To date, neither the precise composition nor the total structure of the $(144,60)$ cluster has been determined by crystallographic methods. The nonaqueous analog stood out in low resolution $(\sim 1 \mathrm{k})$ laser desorption mass spectra in $1995^{28}$ when it was known to some as ubiquitous $29 \mathrm{kDa}$ or (core mass) $\mathrm{Au}_{145}$. Initial analysis by ESI-MS hinted at a composition of $(144,59)^{27}$ soon revised to $(144,60) .{ }^{13,31,37}$ Theoretically, its structure was interpreted as an $\mathrm{Au}_{114}$ icosahedral core mantled in thirty $(1,2)$ units. ${ }^{37-38}$ Presently, we are able to study the aqueous cluster at a much higher resolution of $17 \mathrm{k}$.

Contrary to the $\mathrm{Au}_{102}$-fraction, sodium is not the abundant adduct in the $\mathrm{Au}_{144}$-rich fraction. Instead, water and another species with $\sim 64 \mathrm{Da}$ mass are the two dominant adducts. Their presence was previously used beneficially to assign charge states in the lower resolution spectra ${ }^{17}$ and to discriminate spurious signals (that lack such satellite peaks) from those associated with other gold fractions components ( $35 \mathrm{kDa}, 35.4 \mathrm{kDa}$, and $45 \mathrm{kDa}$, for instance). Evidence for the presence and identification of these adducts is presented in the supplemental section.

The heavier fraction yielded a spectra dominated by $z=5$ - to 9 - states of $(143,58)$, the assigned fragment product of $(144,60)$. It was possible to detect the intact $(144,60)$ species, albeit with lower intensities than the fragment ion. Also present, were identifiable contributions from $(102,44)$ and $(130,50),(137,56)$, and $(180,66)$. The presence of $(130,50)$ was previously suspected by the detection of dodecahedral structure during microscopy analysis (see Supplementary Information) but could not be verified by mass spectrometry until now.

Consistent with the fragmentation pattern observed for $(102,44)$, the icosahedral $(144,60)$ cluster also lost a $(1,2)$ unit while reducing its charge during the ESI process, per the following scheme

$$
(\mathbf{1 4 4 , 6 0})^{[(\mathbf{z}+1)-]} \rightarrow(\mathbf{1 4 3}, \mathbf{5 8})^{[\mathbf{z}-]}+(\mathbf{1 , 2})^{[-]}
$$

The presence of the $(130,50)$ cluster is clearly demonstrated by a series of [ $z=5-$ to 9-] signals in the mass spectra of the $\mathrm{Au}_{144}$ rich fraction (Figures 1 and 3 ). A unique feature of this cluster is that its signal is dominated by its intact structure, an observation that may be interpreted as proof of its unique stability.

Collisional induced dissociation (CID) of the $\mathrm{Au}_{144}$ fraction both at the source and in a high energy cell of the mass spectrometer reveals the resilience of $(130,50)$, as summarized in Figure 5. The striking fact that the $(130,50)^{[z]}$ species was detected at these conditions while $(144,60)$ and others were not, indicates that it is indeed an exceptionally stable species. Its resilience suggest that it may be enriched by chemical etching of the more abundant parent species as has been demonstrated by the nonaqueous counterpart ${ }^{39}$ and explored at harsher conditions than other clusters. 
To the best of our knowledge, ours is the first direct mass spectrometric evidence of the existence and stability of the aqueous analog of the (130,50) cluster, although we are aware that its presence has been suspected from electrophoretic separations of aqueous fractions. ${ }^{40}$ The existence of the nonaqueous analog has been amply characterized by mass spectrometry, ${ }^{39-41}$ theoretically modeled ${ }^{43}$ and its total structure determined by $\mathrm{x}$-ray diffraction. ${ }^{4}$

The presence of $(1,2)$-dissociation products of $(137,56)$ was also apparent from visual examination of the raw mass spectra (Figure 1) and was corroborated by the protein deconvolution software. The MS-characterization of a nonaqueous analog of isolated $(137,56)$ has been previously reported. ${ }^{45}$ At present, its molecular structure remains unknown.

Closer examination shows at least $z=6$ - to 9 - states corresponding to a species of $\sim 45 \mathrm{kDa}$, and one may identify fragments ascribed tentatively to $(180,66)$. The presence of the latter is inferred from the following proposed fragmentation sequence:

$$
(180,66) \rightarrow(179,64) \rightarrow(178,64) \rightarrow(176,64) \rightarrow(175,62)
$$

Although $(180,66)$ is slightly smaller than the higher generation $(187,68)$ species known ${ }^{42}$ and modelled by others, ${ }^{46}$ the proposed assignments of $(180,66)$ and $(179,64)$ are interesting because if they are built from a central core by adding $(1,2)$ units, then the core would turn out to be a 147-atom regular icosahedron or it's close cuboctahedral analog. Furthermore, the 146-atom core of the $(178,64)$ species in the above series may be related to a Mark's decahedral structure.

\section{DISCUSSION}

This report concerns a rather narrowly defined question of longstanding concern; $32,35,47-48$ namely: What are the precise identities of the aqueous gold thiolate clusters, in the range of $\sim 100-200 \mathrm{Au}$ atoms $(\sim 1.5-2.0 \mathrm{~nm}$ in equivalent core-diameter; $20-40 \mathrm{kDa}$ core mass), that are generated in abundance during the reductive decomposition of small anionic $\mathrm{Au}$ $p M B A$ complexes, where $p \mathrm{MBA}=p$-mercaptobenzoate $\left(-\mathrm{S}-\mathrm{Ph}-\mathrm{CO}_{2}{ }^{-}\right)$?

Of particular interest is the region near the special composition $(145,60)^{*}$, which has long been associated with the chiral-icosahedral structure. ${ }^{37} \mathrm{~A}$ key question is how similar are these to the nonaqueous systems, i.e. those formed from hydrophobic thiolate $(R S$-) rest groups $(R)$, and especially when those $R$-groups are sterically demanding and/or allaromatic in structure and bonding.

The technical difficulties preventing an earlier solution to this problem are made clear (above) in the course of the description of our approach to circumventing them ${ }^{17}$ and our proposed answer, with certain caveats, may be stated in these simple terms:

High-resolution ESI-MS can be used to demonstrate positively that this region is populated by the compositions, denoted $\operatorname{Au}_{\mathrm{n}}(p \mathrm{MBA})_{\mathrm{p}}=(\mathrm{n}, \mathrm{p})$, of $(144,60),(137,56)$, and $(130,50)$, in the respective ratios of circa $4: 2: 1$, wherein these compositions appear to be directly 
analogous to the pure compounds established for nonaqueous (and non-aromatic) R-groups. A key step in this work was the analysis of pure aqueous $(102,44)$, of established structure, at isotopic resolution $(\mathrm{m} / \Delta \mathrm{m}>>30,000)$, whereby the efficient dissociation mechanism of $(1,2)$ loss could be identified. This elucidation of the $(1,2)$ coulombic fragmentation pathway is made conclusive by virtue of the great breakthrough in the total-structure determination of the $\mathrm{Au}_{102}(p \mathrm{MBA})_{44}$ cluster. $^{35}$

More recent developments indicated key distinctions between the aliphatic and aromatic ligands: at 21-kDa core mass, the main aliphatic composition was $(104,45)$ of $A u-p e t h$, (peth $=$ phenylethanethiol, $\mathrm{SCH} 2 \mathrm{CH} 2 \mathrm{Ph})^{49}$ rather than $(102,44)$ of $A u-p \mathrm{MBA}$. At low mass, the Price-like compounds of Au-SPh* were shown to be compounds like (36,24), and an extended series derived therefrom by multiples of $\pm(8,4) .{ }^{50}$ Total-structure determinations confirmed that quite different bonding elements were present. A determination of the structure of the $\sim 14-\mathrm{kDa} \mathrm{Au}-p \mathrm{MBA}$ compound ( $\mathrm{Au}_{68}$, vs. $\mathrm{Au}_{67}$ aliphatic) also suggested nonclassical elements in the ligand coordination. ${ }^{18}$ It remains partially uncertain whether the primary cause of these differences is the steric vs. electronic (conjugation) factors. As demonstrated by Bakr and colleagues, the nature of the protecting ligand may affect the size and structure of a cluster. ${ }^{51}$ That solvent (water) effects are less important is indicated by the recent work of Dass, Fortunelli \& coworkers on the hydrophobic $\mathrm{Au}_{102}(\mathrm{SPh})_{44}$ compound $^{52}$.

The persistent intractability of the larger $(n>40)$ aqueous aromatic gold-thiolate compounds to structure- and composition-determination has remained almost until the present moment, even as they have enjoyed ever wider applicability, particularly in the bioconjugation and medical arenas. ${ }^{53,54}$

Tvedte and Ackerson ${ }^{55}$ employed the 'size-focusing' (thermodynamic) approach to identify three (3) super-stable species, tentatively assigned to compositions $(102,44),(144,60)$ and ( 288), with insignificant quantities of intermediate sizes remaining. Mustalahti et al. ${ }^{40}$ purified an intermediate species, to which by comparison to properties of nonaqueous (nonaromatic) $\mathrm{Au}_{130}(\mathrm{SR})_{50}$, of known structure, they associated the composition numbers $(130,50)$.

Finally, very recently, Billinge, Ackerson \& coworkers ${ }^{56}$ have provided extensive X-ray scattering evidence for polymorphism (competing icosahedral and decahedral motifs) in the (nominally) $\mathrm{Au}_{144}(\mathrm{SR})_{60}$ compounds, especially when the $\mathrm{RS}^{-}=p \mathrm{MBA}$ ligand is used. And Plascencia et al. ${ }^{17}$ have reported ESI MS evidence that this $(144,60)$ composition is indeed present and dominant in the PAGE separated samples previously designated by this appellation.

The existence of the multiple species detected along with a putative $(144,60)$ fraction points out the need for further purification efforts that when successful will allow the production of pure $(144,60)$ and confirmation of the celebrated chiral icosahedral structure of relevance in areas ranging from bioconjugation chemistry ${ }^{57}$ to virology. ${ }^{58} \mathrm{~A}$ further stage of this research, well underway, is to eliminate adduct formation in the electrospray process, and to employ inline LCMS methods. ${ }^{59}$ 
As was already apparent in the analytical ultracentrifugation (AUC) results reported earlier by Plascencia et al. ${ }^{17}$ heterogeneity existed within the major three peaks reported in the unpurified mixtures of AuNPs. The presence of additional species in the central fraction $\mathrm{Au}_{144}$ could be further verified by measuring this purified fraction under optimal conditions with higher centrifugal forces in an instrument optimized for hydrodynamic rather than spectral resolution. This would provide additional detail to help identify the other species present in the purified fraction. By studying the purified fraction in isolation, all signal will come from the species of interest, which may have been lost in the multi-wavelength AUC instrument under low concentration conditions. Higher rotor speeds will enhance the sedimentation signal and suppress the diffusion signal, further separating different species with similar hydrodynamic properties. The capabilities of AUC techniques for elucidating particle size and density distributions has been amply demonstrated by Bakr and collaborators. ${ }^{60}$

In our previous report, ${ }^{17}$ we employed advanced electron microscopy (aberration-corrected STEM) to effectively identify the dominant icosahedral structure-type, in this same material, which is consistent with the refined structure-model of Bahena et al. ${ }^{38}$ However, also among the collected HREM images are those indicating the coexistence of minority component of the truncated decahedral, FCC or decahedral structure-type with similar sizes $(\sim 2 \mathrm{~nm}$, Supplementary Information), which may agree with modelled $\mathrm{Au}_{146-147}$ or the recently published total structure determination of $\mathrm{Au}_{130}$ by Chen et al. ${ }^{44} \mathrm{Au}_{130}$ may result from reorganization of $\mathrm{Au}_{144}$ induced by etching of thiolated compounds removing accessible $\mathrm{Au}$ atoms. Figure 6 displays a comparison of the different structural organization of these clusters, assuming that the $(130,50)$ structure remains the same as the decahedral one, reported in its total structure determination. ${ }^{28}$ This comparison indicates that not only the icosahedral $\mathrm{Au}_{114}$ grand core is reorganized into a decahedral $\mathrm{Au}_{105}$ grand core, but also the $(130,50)$ ligand shell loses 5 monomeric "staple" motifs. Interestingly, this re-organization does not modify the chirality of these clusters that is mainly due to chiral arrangement of the "staple motifs" covering a grand core with a small index of chirality. ${ }^{61}$

Further results with detailed analysis and characterization will be reported separately, along with results obtained using the rapid scanning electron diffraction method developed by Bahena et al. ${ }^{38}$ and Bruma et al. ${ }^{19}$

Here we have emphasized these points, because the reliable and efficient determination of the composition of mixtures of similar size clusters is the sine qua non of the comprehensive application of chemical thermodynamics to these intriguing systems on the borderline between molecular and macroscopic phases. The aqueous Au- $p$ MBA system is especially attractive for such thermochemical investigations, and in particular to probe the NanocrystalMicroemulsion picture of Gelbart et al..$^{25}$ because all components exist always as soluble species in a single (aqueous) reaction system. The Orbitrap extended mass range platform has hereby been demonstrated to have sufficient sensitivity and resolution to accomplish these objectives. 


\section{CONCLUDING REMARKS}

Herein we have described work aimed at removing the barriers to high resolution determination of the intact aqueous cluster-compound(s) present in such samples. Specifically, the results described here support the view that there exists a series of thermodynamically favored sizes and structures common to both aqueous and nonaqueous systems. The aqueous clusters protected with $p \mathrm{MBA}$, preferably fragment by reducing their charge state and expelling $(1,2)$ anionic units.

High resolution mass spectrometry reveals that despite extensive purification efforts via PAGE, the $(144,60)$ fraction is accompanied by notable byproducts including $(102,44)$, $(130,50)$, and $(137,56)$. Of these, $(130,50)$ outstands by its resilience to collisional induced dissociation, a feature that points at its stability. Our results also show evidence for the presence of a novel higher mass species, tentatively assigned a composition of $(180,66)$. A protocol for improving future purifications by cation exchange with a tertiary ammonium cation is proposed to aid future purification efforts towards the isolation of pure $(144,60)$ crystals for total structure determination.

Beyond the immediate objectives -- to identify the compositions present, and to compare those to the ones well established from nonaqueous \& aliphatic Au-SR research -- the ability to determine rapidly the relative abundances obtained from various preparations as a function of thermochemical variables (concentrations, $\mathrm{pH}$, electrochemical potential, temperature) should allow, via the thermodynamic theory (nano-crystal-microemulsion picture) to determine the critical differences among the species present.

\section{Supplementary Material}

Refer to Web version on PubMed Central for supplementary material.

\section{ACKOWLEDGMENTS}

We thank Dr. J. J. Pelayo for his assistance in the visualization of the cluster theoretical structures. This work has been supported by The Welch Foundation (AX-1615 and AX-1857), NSF-DAC (1339649), NSF (TGMCB-070039), and Texas-Stars Foundation. Facilities of NIH RCMI Nanotechnology and Human Health Core (RCMI Grant 5G12RR013646-12) and NIH RCMI Biophotonics (RCMI Grant G12MD007591) at UTSA. UTSA authors acknowledge generous support form Thermo Fisher Scientific for providing access to the Orbitrap-EMR instrument.

\section{ABBREVIATIONS:}

$\begin{array}{ll}(\mathbf{n}, \mathbf{p}) & \mathrm{Au}_{\mathrm{n}}(p \mathrm{MBA})_{\mathrm{p}} \\ \text { ESI-MS } & \text { electron spray ionization mass spectrometry } \\ \text { CID } & \text { collisional induced dissociation } \\ p \text { MBA } & p \text {-mercaptobenzoic acid } \\ \text { Peth } & \text { phenylethanethiol, } \mathrm{SCH}_{2} \mathrm{CH}_{2} \mathrm{Ph} .\end{array}$




\section{REFERENCES}

(1). Faraday M, The Bakerian Lecture: Experimental Relations of Gold (and Other Metals) to Light. Philos. Trans. R. Soc. London 1857, 147, 145-181.

(2). Edwards PP; Thomas JM, Gold in a Metallic Divided State-from Faraday to Present-Day Nanoscience. Angew. Chem. Int. Ed 2007, 46, 5480-5486.

(3). Dass A, Faradaurate Nanomolecules: A Superstable Plasmonic 76.3 Kda Cluster. J. Am. Chem. Soc 2011, 133, 19259-19261. [PubMed: 22077797]

(4). Tsukuda T, Toward an Atomic-Level Understanding of Size-Specific Properties of Protected and Stabilized Gold Clusters. Bull. Chem. Soc. Jpn 2012, 85, 151-168.

(5). Negishi Y; Nakazaki T; Malola S; Takano S; Niihori Y; Kurashige W; Yamazoe S; Tsukuda T; Häkkinen H, A Critical Size for Emergence of Nonbulk Electronic and Geometric Structures in Dodecanethiolate-Protected Au Clusters. J. Am. Chem. Soc 2015, 137, 1206-1212. [PubMed: 25549276]

(6). Alvarez MM; Khoury JT; Schaaff TG; Shafigullin MN; Vezmar I; Whetten RL, Optical Absorption Spectra of Nanocrystal Gold Molecules. J. Phys. Chem. B 1997, 101, 3706-3712.

(7). Wyrwas RB; Alvarez MM; Khoury JT; Price RC; Schaaff TG; Whetten RL, The Colours of Nanometric Gold. Eur. Phys. J. D 2007, 43, 91-95.

(8). Schaaff TG; Whetten RL, Giant Gold-Glutathione Cluster Compounds: Intense Optical Activity in Metal Based Transitions. J. Phys. Chem. B 2000, 104, 2630-2641.

(9). Qian H; Zhu Y; Jin R, Atomically Precise Gold Nanocrystal Molecules with Surface Plasmon Resonance. Proc. Natl. Acad. Sci. USA 2012, 109, 696-700. [PubMed: 22215587]

(10). Pichugina DA; Kuz'menko NE; Shestakov AF, Ligand Protected Gold Clusters: The Structure, Synthesis and Applications. Russ. Chem. Rev 2015, 84, 1114-1144.

(11). Kurashige W; Niihori Y; Sharma S; Negishi Y, Precise Synthesis, Functionalization and Application of Thiolate-Protected Gold Clusters. Coord. Chem. Rev 2016 DOI: 10.1016/j.ccr. 2016.02.013

(12). Chaki NK; Negishi Y; Tsunoyama H; Shichibu Y; Tsukuda T, Ubiquitous 8 and 29 kDa Gold:Alkanethiolate Cluster Compounds: Mass Spectrometric Determination of Molecular Formulas and Structural Implications. J. Am. Chem. Soc 2008, 130, 8608-8610. [PubMed: 18547044]

(13). Fields Zinna CA; Sardar R; Beasley CA; Murray RW, Electrospray Ionization Mass Spectrometry of Intrinsically Cationized Nanoparticles, $\left[\mathrm{Au}_{144} /{ }_{146}\left(\mathrm{SC}_{11} \mathrm{H}_{22} \mathrm{n}\left(\mathrm{CH}_{2} \mathrm{CH}_{3}\right)_{3}\right) \times\right.$

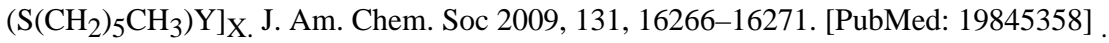

(14). Qian H; Jin R, Controlling Nanoparticles with Atomic Precision: The Case of $\mathrm{Au}_{144}\left(\mathrm{SCH}_{2} \mathrm{CH}_{2} \mathrm{Ph}\right)_{60}$. Nano Lett. 2009, 9, 4083-4087. [PubMed: 19995083]

(15). Dreaden EC; Alkilany AM; Huang X; Murphy CJ; El-Sayed MA, The Golden Age: Gold Nanoparticles for Biomedicine. Chem. Soc. Revv 2012, 41, 2740-2779.

(16). Louis C; Pluchery O, Gold Nanoparticles for Physics, Chemistry and Biology. World Scientific: 2012.

(17). Plascencia Villa G; Demeler B; Whetten RL; Griffith WP; Alvarez M; Black DM; José-Yacamán M, Analytical Characterization of Size-Dependent Properties of Larger Aqueous Gold Nanoclusters. J. Phys. Chem. C 2016, 120, 8950-8958.

(18). Azubel M; Koivisto J; Malola S; Bushnell D; Hura GL; Koh AL; Tsunoyama H; Tsukuda T; Pettersson M; Hkkinen H, Electron Microscopy of Gold Nanoparticles at Atomic Resolution. Science 2014, 345, 909-912. [PubMed: 25146285]

(19). Bruma A; Santiago U; Alducin D; Plascencia Villa G; Whetten RL; Ponce A; Mariscal MM; Jose Yacaman M, Structure Determination of Superatom Metallic Clusters Using Rapid Scanning Electron Diffraction. J. Phys. Chem. C 2016, 120, 1902-1908

(20). Chen F; Gülbakan B; Weidmann S; Fagerer SR; Ibáñez AJ; Zenobi R, Applying Mass Spectrometry to Study Non-Covalent Biomolecule Complexes. Mass Spectrom. Rev 2016, 35, 48-70. [PubMed: 25945814] 
(21). Hare DJ; New EJ, On the Outside Looking In: Redefining the Role of Anal. Chem. in the Biosciences. Chem. Comun 2016, In print.

(22). Rose RJ; Damoc E; Denisov E; Makarov A; Heck AJR, High-Sensitivity Orbitrap Mass Analysis of Intact Macromolecular Assemblies. Nat. Methods 2012, 9, 1084-1086. [PubMed: 23064518]

(23). Wang Y; Herron N, Nanometer-Sized Semiconductor Clusters: Materials Synthesis, Quantum Size Effects, and Photophysical Properties. J. Phys. Chem 1991, 95, 525-532.

(24). Herron N; Suna A; Wang Y, Synthesis of $\approx 10 \AA$ Thiophenolate-Capped CdS Clusters. Observation of a Sharp Absorption Peak. J. Chem. Soc., Dalton Trans 1992, 2329-2335.

(25). Whetten RL; Gelbart WM, Nanocrystal Microemulsions: Surfactant-Stabilized Size and Shape. J. Phys. Chem 1994, 98, 3544-3549.

(26). Leff DV; Ohara PC; Heath JR; Gelbart WM, Thermodynamic Control of Gold Nanocrystal Size: Experiment and Theory. J. Phys. Chem 1995, 99, 7036-7041.

(27). Jin R; Qian H; Wu Z; Zhu Y; Zhu M; Mohanty A; Garg N, Size Focusing: A Methodology for Synthesizing Atomically Precise Gold Nanoclusters. J. Phys. Chem. Lett 2010, 1, 2903-2910.

(28). Brust M; Walker M; Bethell D; Schiffrin DJ; Whyman R, Synthesis of Thiol Derivatised Gold Nanoparticles in a Two-Phase Liquid-Liquid System. J. Chem. Soc., Chem. Commun 1994, 801802.

(29). Whetten RL; Khoury JT; Alvarez MM; Murthy S; Vezmar I; Wang ZL; Stephens PW; Cleveland CL; Luedtke WD; Landman U, Nanocrystal Gold Molecules. Adv. Mater 1996, 8, 428-433.

(30). Terrill RH; Postlethwaite TA; Chen C.-h.; Poon C-D; Terzis A; Chen A; Hutchison JE; Clark MR; Wignall G Monolayers in Three Dimensions: NMR, SAXS, Thermal, and Electron Hopping Studies of Alkanethiol Stabilized Gold Clusters. J. Am. Chem. Soc 1995, 117, 12537-12548.

(31). Schaaff TG; Shafigullin MN; Khoury JT; Vezmar I; Whetten RL, Properties of a Ubiquitous 29 kDa Au: SR Cluster Compound. J. Phys. Chem. B 2001, 105, 8785-8796.

(32). Negishi Y; Nobusada K; Tsukuda T, Glutathione Protected Gold Clusters Revisited: Bridging the Gap between Gold (I)-Thiolate Complexes and Thiolate-Protected Gold Nanocrystals. J. Am. Chem. Soc 2005, 127, 5261-5270. [PubMed: 15810862]

(33). Price RC; Whetten RL, All-Aromatic, Nanometer Scale, Gold Cluster Thiolate Complexes. J. Am. Chem. Soc 2005, 127, 13750-13751. [PubMed: 16201770]

(34). Ackerson CJ; Jadzinsky PD; Kornberg RD, Thiolate Ligands for Synthesis of Water-Soluble Gold Clusters. J. Am. Chem. Soc 2005, 127, 6550-6551. [PubMed: 15869273]

(35). Jadzinsky PD; Calero G; Ackerson CJ; Bushnell DA; Kornberg RD, Structure of a Thiol Monolayer Protected Gold Nanoparticle at 1.1 a Resolution. Science (New York, N.Y.) 2007, 318, 430-433. [PubMed: 17947577]

(36). Levi-Kalisman Y; Jadzinsky PD; Kalisman N; Tsunoyama H; Tsukuda T; Bushnell DA; Kornberg $\mathrm{RD}$, Synthesis and Characterization of $\mathrm{Au}_{102}$ (P MBA) 44 Nanoparticles. J. Am. Chem. Soc 2011, 133, 2976-2982. [PubMed: 21319754]

(37). Lopez-Acevedo O; Akola J; Whetten RL; Gronbeck H; Häkkinen H, Structure and Bonding in the Ubiquitous Icosahedral Metallic Gold Cluster $\mathrm{Au}_{144}(\mathrm{SR})_{60}$. J. Phys. Chem. C 2009, 113, 5035-5038.

(38). Bahena D; Bhattarai N; Santiago U; Tlahuice A; Ponce A; Bach SBH; Yoon B; Whetten RL; Landman U; Jose Yacaman M, STEM Electron Diffraction and High-Resolution Images Used in the Determination of the Crystal Structure of the $\mathrm{Au}_{144}(\mathrm{SR})_{60}$ Cluster. J. Phys. Chem. Lett 2013, 4, 975-981. [PubMed: 23687562]

(39). Jupally VR; Dass A, Synthesis of $\mathrm{Au}_{130}(\mathrm{SR})_{50}$ and $\mathrm{Au}_{130}-\mathrm{XAgX}(\mathrm{SR})_{50}$ Nanomolecules through Core Size Conversion of Larger Metal Clusters. Phys. Chem. Chem. Phys 2014, 16, 10473-10479. [PubMed: 24733419]

(40). Mustalahti S; Myllyperkiö P; Lahtinen T; Malola S; Salorinne K; Tero T-R; Koivisto J; Häkkinen $\mathrm{H}$; Pettersson M, Photodynamics of a Molecular Water Soluble Nanocluster Identified as $\mathrm{Au}_{130}(\mathrm{pMBA})_{50}$. J. Phys. Chem. C 2015, 119, 20224-20229.

(41). Tang Z; Robinson DA; Bokossa N; Xu B; Wang S; Wang G, Mixed Dithiolate Durene-Dt and Monothiolate Phenylethanethiolate Protected $\mathrm{Au}_{130}$ Nanoparticles with Discrete Core and Core Ligand Energy States. J. Am. Chem. Soc 2011, 133, 16037-16044. [PubMed: 21919537] 
(42). Negishi Y; Sakamoto C; Ohyama T; Tsukuda T, Synthesis and the Origin of the Stability of Thiolate Protected $\mathrm{Au}_{130}$ and $\mathrm{Au}_{187}$ Clusters. J. Phys. Chem. Lett 2012, 3, 1624-1628. [PubMed: 26285718]

(43). Tlahuice Flores A; Santiago U; Bahena D; Vinogradova E; Conroy CV; Ahuja T; Bach SBH; Ponce A; Wang G; José-Yacamán M, Structure of the Thiolated $\mathrm{Au}_{130}$ Cluster. J. Phys. Chem. A 2013, 117, 10470-10476. [PubMed: 24004091]

(44). Chen Y; Zeng C; Liu C; Kirschbaum K; Gayathri C; Gil RR; Rosi NL; Jin R, Crystal Structure of Barrel-Shaped Chiral Au 130 (p-MBT) 50 Nanocluster. J. Am. Chem. Soc 2015, 137, 1007610079. [PubMed: 26244606]

(45). Jupally VR; Dharmaratne AC; Crasto D; Huckaba AJ; Kumara C; Nimmala PR; Kothalawala N; Delcamp JH; Dass A, Au 137 (SR) 56 Nanomolecules: Composition, Optical Spectroscopy, Electrochemistry and Electrocatalytic Reduction of $\mathrm{CO}_{2}$. Chem. Comun 2014, 50, 9895-9898.

(46). Tlahuice Flores A, New Insight into the Structure of Thiolated Gold Clusters: A Structural Prediction of the $\mathrm{Au}_{187}(\mathrm{SR})_{68}$ Cluster. Phys. Chem. Chem. Phys 2015, 17, 5551-5555. [PubMed: 25640209]

(47). Tracy JB; Crowe MC; Parker JF; Hampe O; Fields-Zinna CA; Dass A; Murray RW, Electrospray Ionization Mass Spectrometry of Uniform and Mixed Monolayer Nanoparticles: $\mathrm{Au}_{25}\left[\mathrm{~S}\left(\mathrm{CH}_{2}\right)_{2} \mathrm{Ph}\right]_{18}$ and $\mathrm{Au}_{25}\left[\mathrm{~S}\left(\mathrm{CH}_{2}\right)_{2} \mathrm{Ph}\right]_{18} \mathrm{X}(\mathrm{SR})_{\mathrm{X}}$. J. Am. Chem. Soc 2007, 129, 16209 16215. [PubMed: 18034488]

(48). Whetten RL; Price RC, Chemistry. Nano Golden Order. Science (New York, NY) 2007, 318, 407-408. [PubMed: 17947573]

(49). Dass A; Nimmala PR; Jupally VR; Kothalawala N, $\mathrm{Au}_{103}(\mathrm{SR})_{45}, \mathrm{Au}_{104}(\mathrm{SR})_{45}, \mathrm{Au}_{104}(\mathrm{SR})_{46}$ and $\mathrm{Au}_{105}(\mathrm{SR})_{46}$ Nanoclusters. Nanoscale 2013, 5, 12082-12085. [PubMed: 24132473]

(50). Zeng C; Chen Y; Iida K; Nobusada K; Kirschbaum K; Lambright KJ; Jin R, Gold Quantum Boxes: On the Periodicities and the Quantum Confinement in the $\mathrm{Au}_{28}, \mathrm{Au}_{36}, \mathrm{Au}_{44}$, and $\mathrm{Au}_{52}$ Magic Series. J. Am. Chem. Soc 2016, 138, 3950-3953. [PubMed: 26934618]

(51). Bootharaju MS; Burlakov VM; Besong TM; Joshi CP; AbdulHalim LG; Black DM; Whetten RL; Goriely A; Bakr OM, Reversible Size Control of Silver Nanoclusters Via Ligand Exchange. Chem. Mater 2015, 27, 4289-4297.

(52). Rambukwella M; Sementa L; Barcaro G; Fortunelli A; Dass A, Organosoluble Au 102 (SPh) 44 Nanomolecules: Synthesis, Isolation, Compositional Assignment, Core Conversion, Optical Spectroscopy, Electrochemistry, and Theoretical Analysis. J. Phys. Chem. C 2015, 119, 2507725084.

(53). Sexton JZ; Ackerson CJ, Determination of Rigidity of Protein Bound $\mathrm{Au}_{144}$ Clusters by Electron Cryomicroscopy. J. Phys. Chem. C 2010, 114, 16037-16042.

(54). Gifford JC; Bresee J; Carter CJ; Wang G; Melander RJ; Melander C; Feldheim DL, Thiol Modified Gold Nanoparticles for the Inhibition of Mycobacterium Smegmatis. Chem. Comun 2014, 50, 15860-15863.

(55). Tvedte LM; Ackerson CJ, Size-Focusing Synthesis of Gold Nanoclusters with pMercaptobenzoic Acid. J. Phys. Chem. A 2014, 118, 8124-8128. [PubMed: 24628255]

(56). Jensen KMØ; Juhas P; Tofanelli MA; Heinecke CL; Vaughan G; Ackerson CJ; Billinge SJL, Polymorphism in Magic Sized $\mathrm{Au}_{144}(\mathrm{SR})_{60}$ Clusters. Nat. Commun 2016 DOI:10.1038/ ncomms11859

(57). Ackerson CJ; Jadzinsky PD; Sexton JZ; Bushnell DA; Kornberg RD, Synthesis and Bioconjugation of 2 and 3 nm Diameter Gold Nanoparticles. Bioconjugate Chem. 2010, 21, 214218.

(58). Zandi R; Reguera D; Bruinsma RF; Gelbart WM; Rudnick J, Origin of Icosahedral Symmetry in Viruses. Proc. Natl. Acad. Sci. USA 2004, 101, 15556-15560. [PubMed: 15486087]

(59). Black DM; Bach SB; Whetten RL, Capillary Liquid Chromatography Mass Spectrometry [LCMS] Analysis of Intact Monolayer-Protected Clusters in Complex Mixtures. Anal. Chem 2016, 88, 5631-5636 [PubMed: 27216373]

(60). Carney RP; Kim JY; Qian H; Jin R; Mehenni H; Stellacci F; Bakr OM, Determination of Nanoparticle Size Distribution Together with Density or Molecular Weight by 2D Analytical Ultracentrifugation. Nat. Commun 2011, 2, 335-343. [PubMed: 21654635] 
(61). Pelayo JJ; Whetten RL; Garzon IL, Geometric Quantification of Chirality in Ligand Protected Metal Clusters. J. Phys. Chem. C 2015, 119, 28666-28678. 


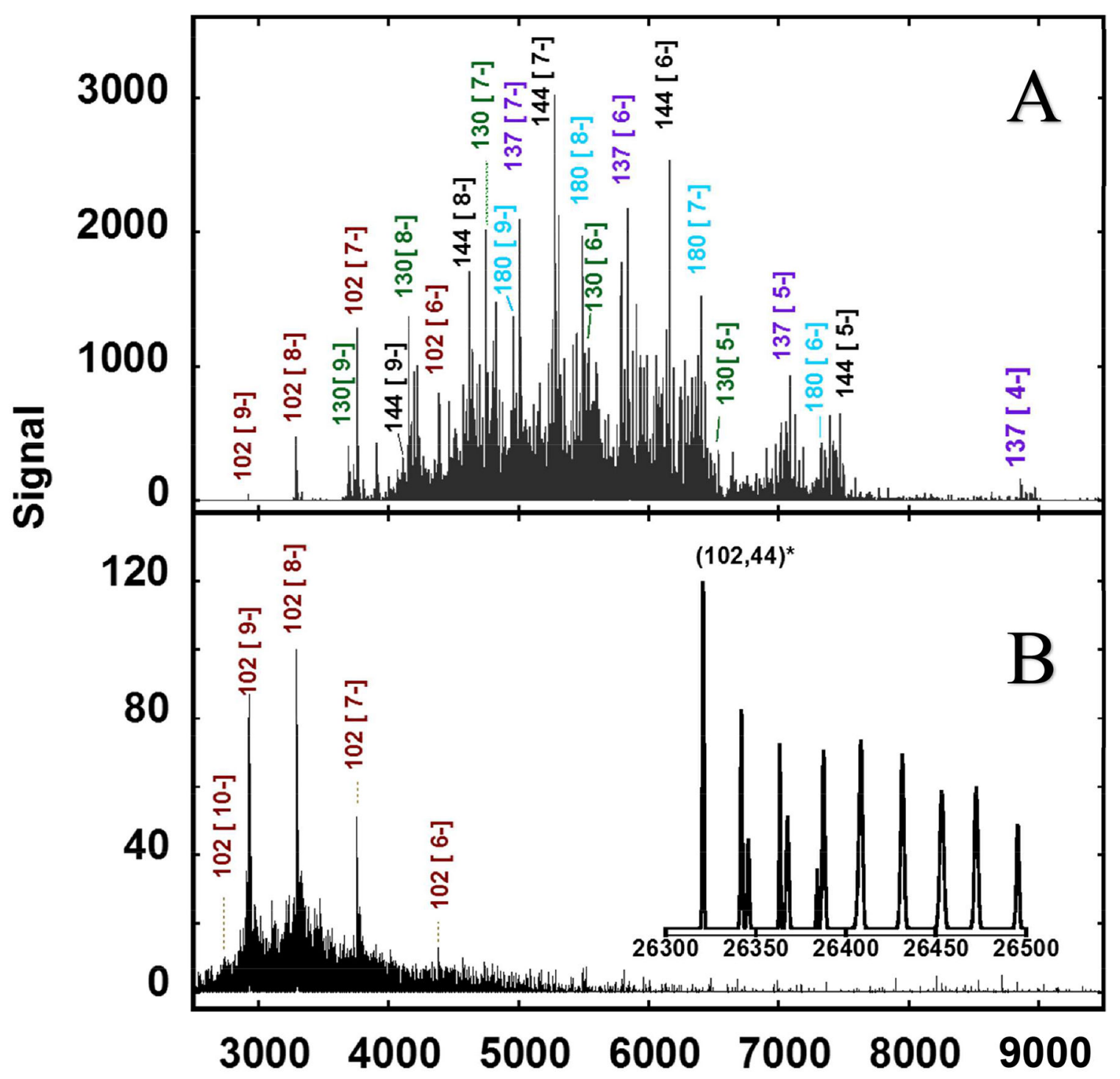

Figure 1: Negative-ion Orbitrap Electrospray-Ionization Mass Spectrometry (ESI-MS), at a resolution $(\mathrm{m} / \Delta \mathrm{m})$ of $17 \mathrm{k}$ for $\mathrm{Au}_{144}$-rich fraction and $43.4 \mathrm{k}$ for the $\mathrm{Au}_{102}$ fraction.

(A) Raw mass spectra of gel-purified $\mathrm{Au}_{144}$-rich fraction showing multiple negatively charged signals $\left[\mathrm{z}=4^{-}\right.$to $\left.8^{-}\right]$from aqueous $(p \mathrm{MBA})$-stabilized $\mathrm{Au}_{\mathrm{N}}$ species $[N=102,130$, 137 , and 180] in addition to the expected $N=144$ signal. Except for $\mathrm{Au}_{130}$, which is dominant, the assigned (indicated) dominant signals correspond to ions produced by loss of one $\mathrm{Au}(\mathrm{pMBA})_{2}{ }^{-}$fragment from the parent species.

(B) Spectra of gel-purified $\mathrm{Au}_{102}$ fraction showing the absence of additional gold species; the multitude of peaks surrounding the base signal is attributed to multiple Na adducts resulting from $\mathrm{Na}$-Hydrogen exchange. The inset shows the deconvolution of the complex raw spectra into a single cluster signal and its $\mathrm{Na}$ adducts using standard deconvolution software. Aqueous solutions were infused at $5 \mu \mathrm{L} / \mathrm{min}$ at a concentration of $\sim 0.5 \mu \mathrm{g} / \mu \mathrm{L}$ and averaged for 5 minutes. 


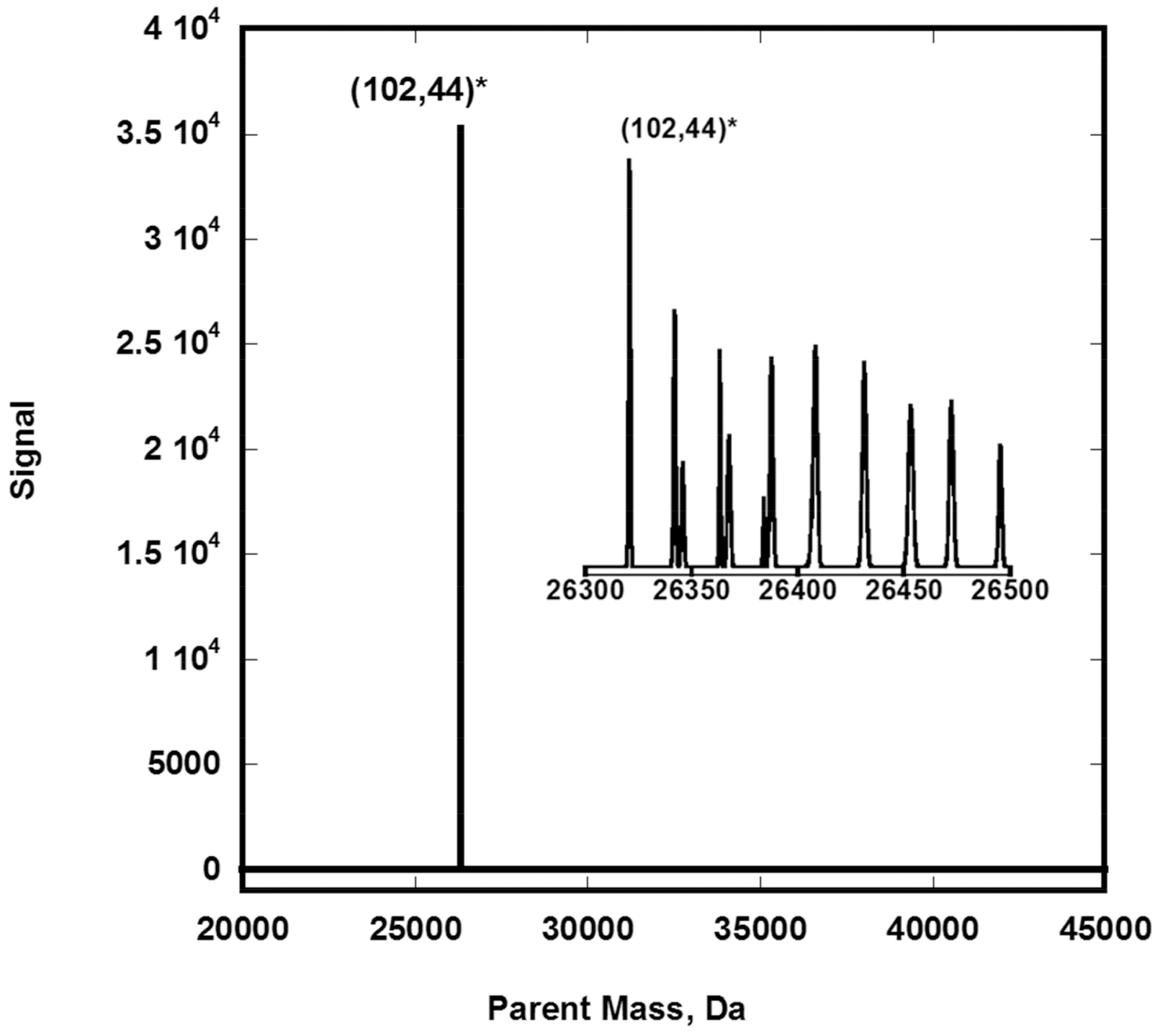

Figure 2: shows how the complex ESIMS spectrogram of the $\mathrm{Au}_{102}$ fraction can be simplified into a single peak using Protein Deconvolution 4.0 software.

The raw negative ion complex spectra of a 50:50 methanol/water solution of the $(102,44)$ fraction was analyzed using Manual Xtract (isotopically resolved) at $4 \mathrm{ppm}$ mass tolerance, $99 \%$ confidence, $2-100 \mathrm{kDa}$ outpout mass range, and intact protein peak model mode. Four charge states where detected $\left(\mathrm{z}=6^{-}\right.$to $\left.9^{-}\right)$. The mass tolerance was relaxed to $20 \mathrm{ppm}$ to detect the sodium adducts depicted in the inset. Infusion rate $=5 \mu \mathrm{L} / \mathrm{min}$; particle concentration $\sim 0.5 \mu \mathrm{g} / \mu \mathrm{L}$; scan duration $=1.0$ minute. 


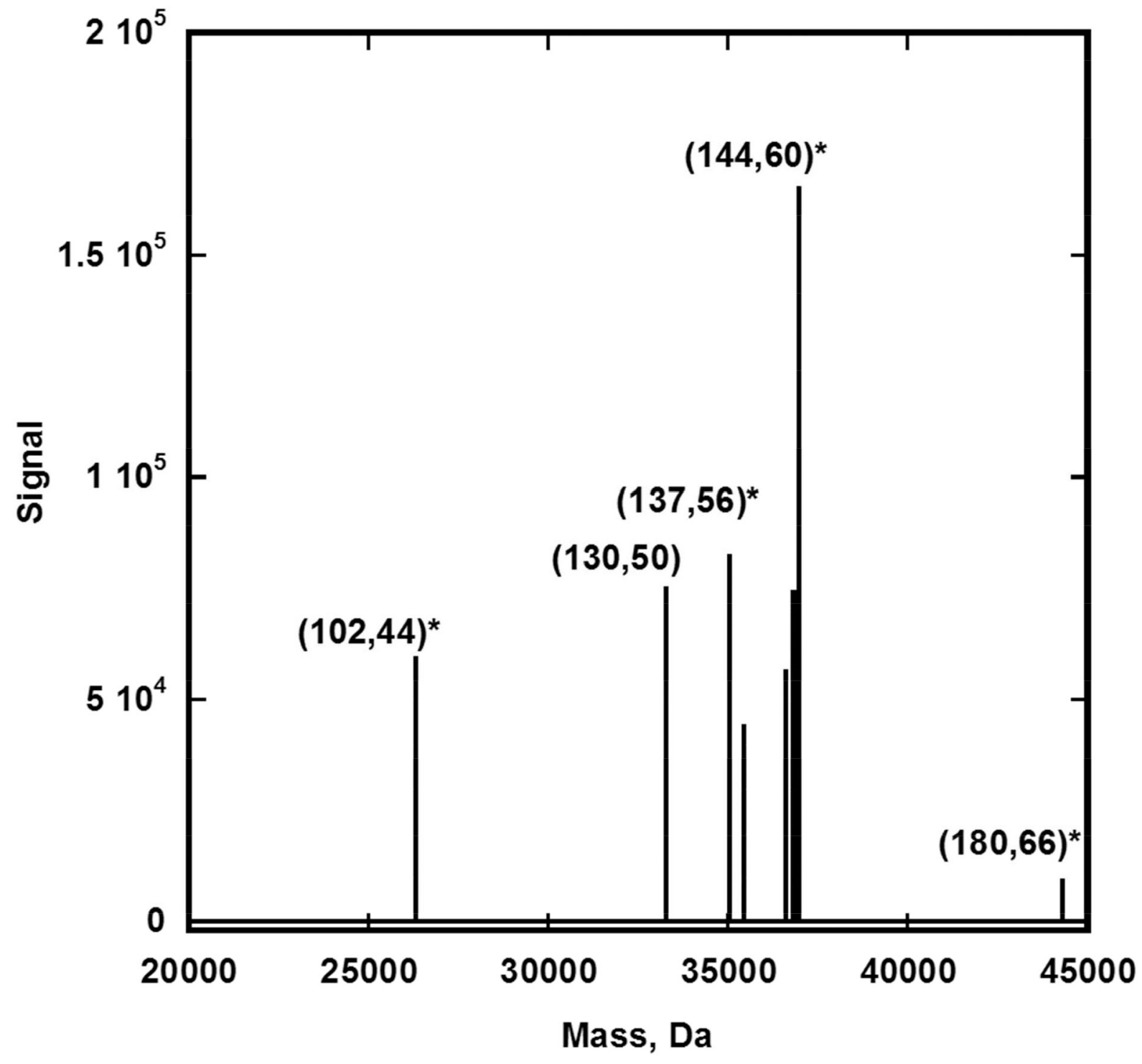

Figure 3: shows the deconvolution of the complex $\mathrm{Au}_{144}$ fraction ESIMS spectrogram into three major components.

The raw negative ion complex spectra of a an aqueous solution of the $(144,60)$ fraction was analyzed using Manual ReSpect ${ }^{\mathrm{TM}}$ (isotopically unresolved) at 5 ppm mass tolerance, $99 \%$ confidence, $2-100 \mathrm{kDa}$ mass range, and intact "protein peak model" mode. Four charge states were detected $(\mathrm{z}=-5$ to -8$)$ by the algorithm. Infusion rate $=5 \mu \mathrm{L} / \mathrm{min}$; particle concentration $\sim 0.5 \mu \mathrm{g} / \mu \mathrm{L}$; scan duration $=4.8$ minutes. Not labelled in the figure are adducts and/or fragment products of $(137,56)[(136,54 X)]$, and $(144,60)[(142,56)$ and $(143,57)]$ 
High Resolution Determination of Four (4) Charge-States of $(102,44)[z=6-$ to $9-]$

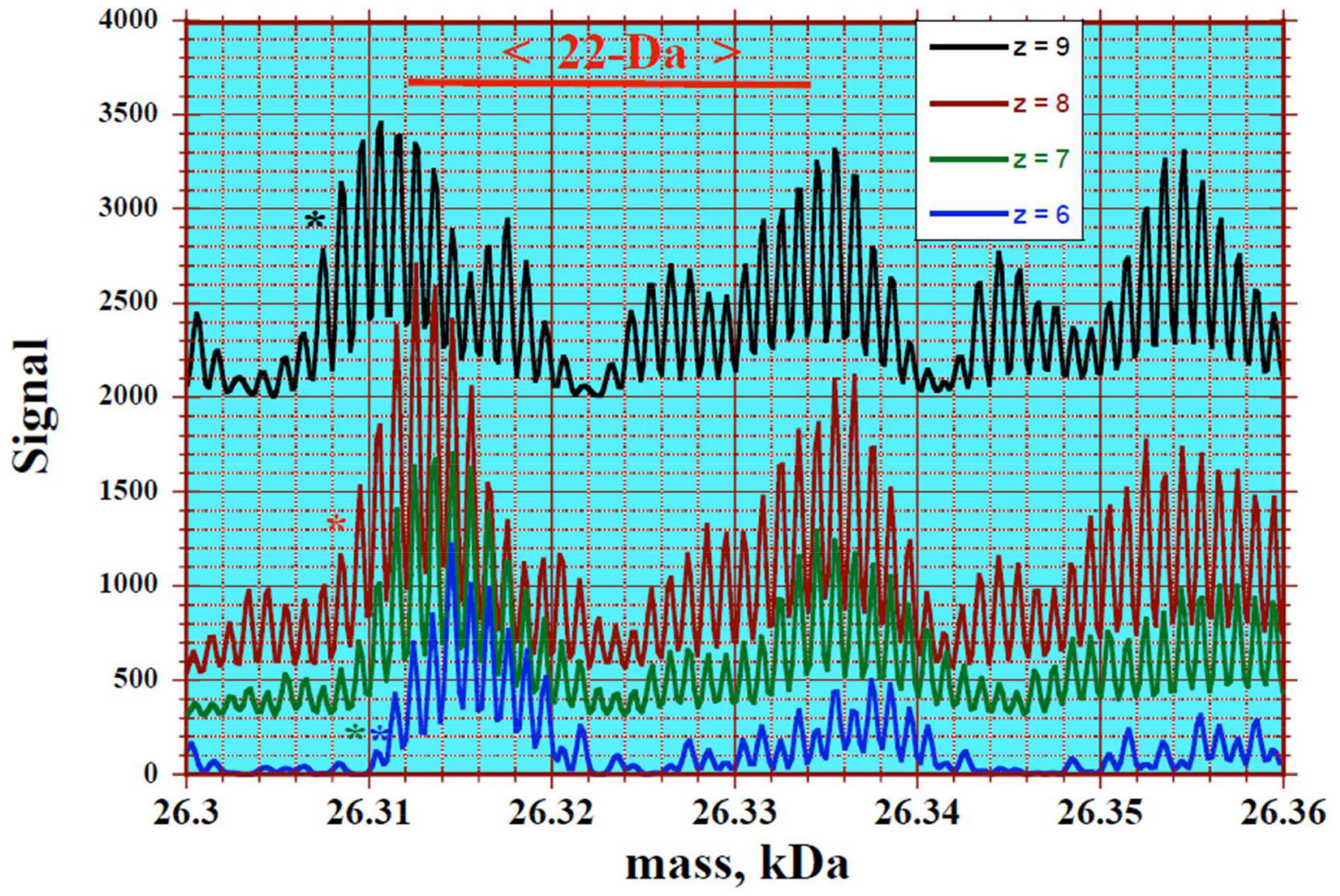

Figure 4: showing the $(101,42)$ fragmentation product and its sodium adduct at isotopic resolution.

Depicted is an overlay of parent masses calculated by multiplying the raw $\mathrm{m} / \mathrm{z}$ signal by the indicated charge (folding). The single-Da peak spacing correspond to isotopic patterns while the larger (22 Da) spacing results from multiple $\mathrm{Na}^{+}$for $\mathrm{H}^{+}$substitutions in the p-MBA ligand. The signal shifts to lower masses upon further ionization by deprotonation. Infusion rate is $5 \mu \mathrm{L} / \mathrm{min}$; particle concentration $\sim 0.5 \mu \mathrm{g} / \mu \mathrm{L}$; scan duration $=1$ minute. 


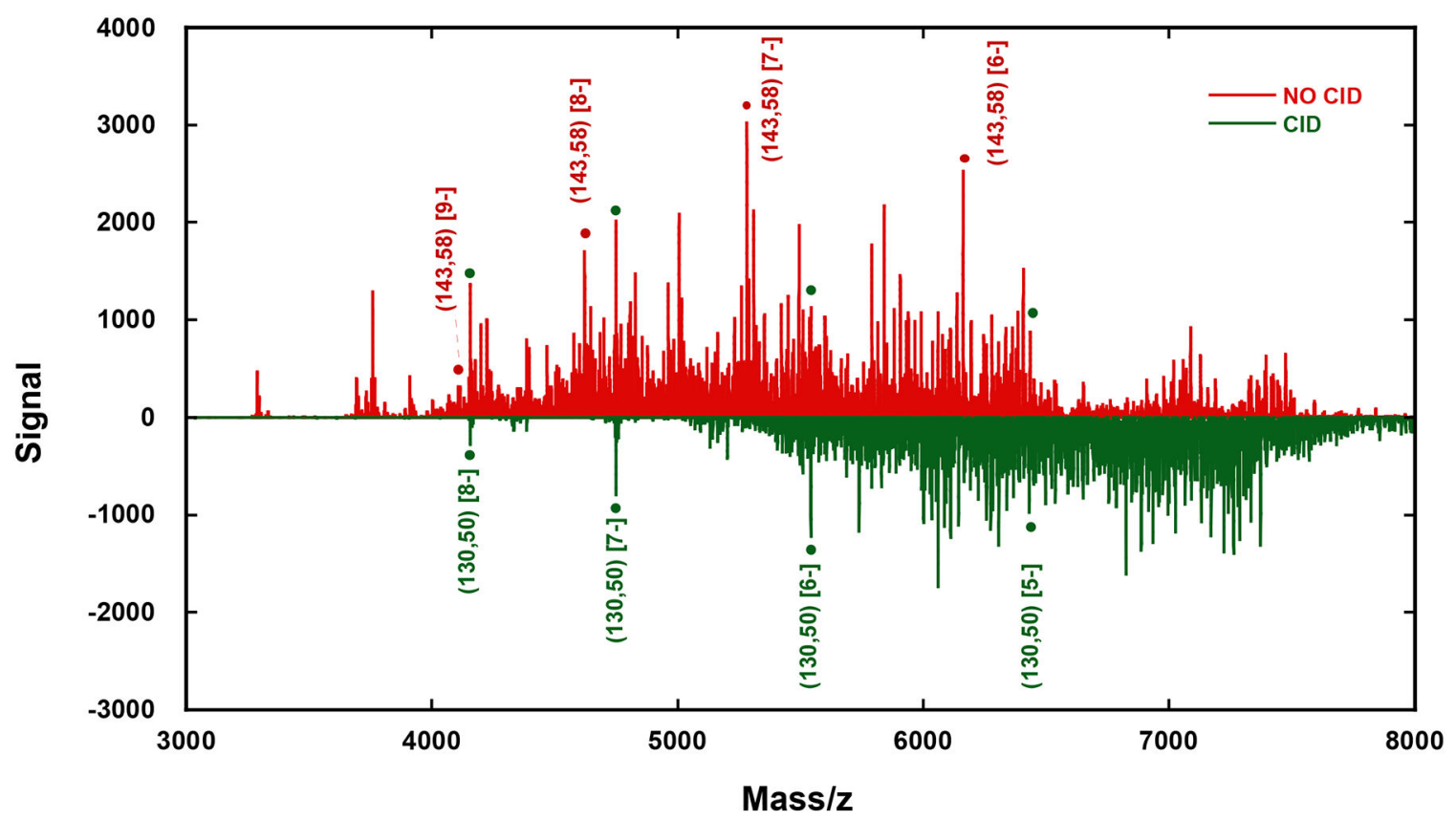

Figure 5: Collisional Dissociation (charge reduction) results demonstrating the resilience of $(130,50)$.

Compared are signals from the aqueous $\mathrm{Au}_{144}$-rich fraction before in source Collisional Induced Dissociation -CID- (positve axis, red trace) and the signal under CID of $20 \mathrm{eV}$ at the source (negative axis, green trace). The signal from (130,50), highlighted with solid green dots, is notable before fragmentation and dominates the low $(\mathrm{m} / \mathrm{z})$-range after CID conditions. Infusion rate is $5 \mu \mathrm{L} / \mathrm{min}$; particle concentration $\sim 0.5 \mu \mathrm{g} / \mu \mathrm{L}$; scan duration $=0.5$ minutes. 


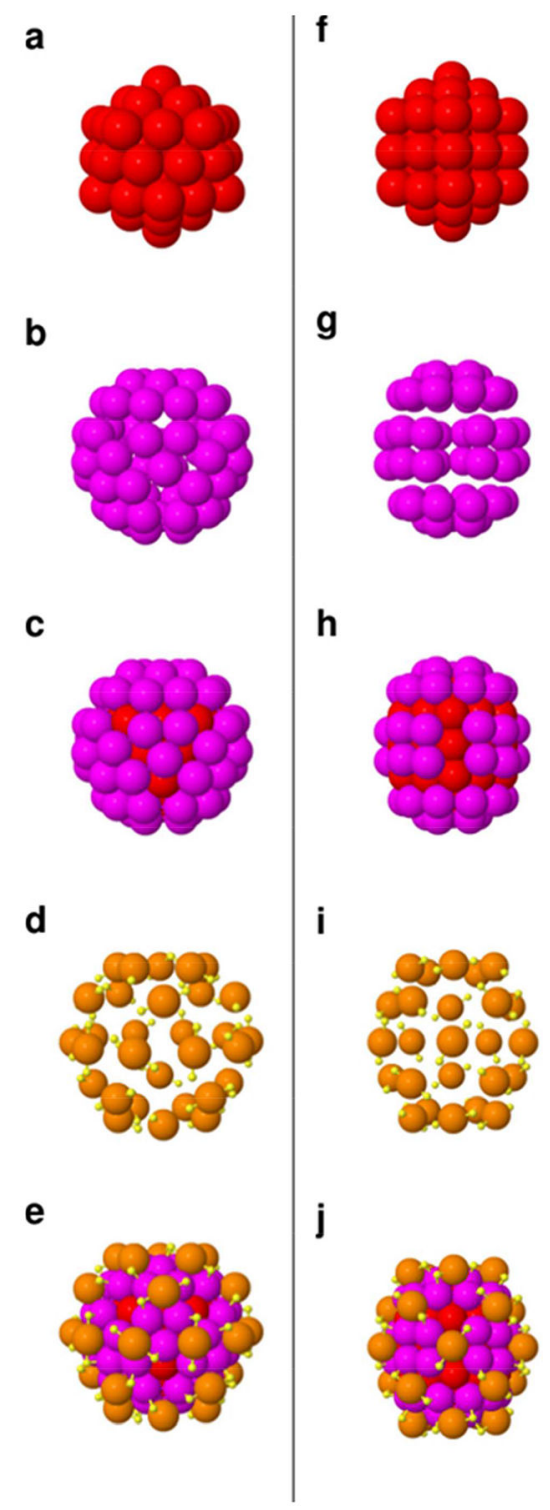

Figure 6. Structure-comparison of the icosahedral $(144,60)$ and truncated decahedral $(130,50)$ clusters.

Side-by-side scaled comparison of the structures of the $(144,60)$ (LHS) and $(130,50)$ (RHS) clusters, omitting only the R-groups.

(a) Fifty-four atom gold core consisting of two Mackay icosahedron shells. (b) 60-atom gold shell. (c) The icosahedral gold core (red) is covered by 60 gold atoms (pink) making up the $\mathrm{Au}_{114}$ grand core. (d) 30 monomeric "staple" motifs (yellow atoms represents sulfur, whereas orange atoms correspond to gold atoms in the "staple" motifs). (e) Total structure of the icosahedral $\mathrm{Au}_{144}(\mathrm{SR})_{60}$ cluster, where the grand core is covered with 30 "staple" motifs. (f) Fifty-five atom gold core in a decahedral structure. (g) 50-atom gold shell. (h) The decahedral gold core (red) is covered by the 50 -atom shell (pink) making up the $\mathrm{Au}_{105}$ grand core. (i) 25 monomeric "staple" motifs (yellow atoms represents sulfur, whereas orange atoms correspond to gold atoms in the "staple" motifs). (j) Total structure of the decahedral $\mathrm{Au}_{130}(\mathrm{SR})_{50}$ cluster, where the grand core is covered with 25 "staple" motifs. 
Perspectives are as follows: In both cases, the structures are oriented such that a C5-axis lies vertical (in the plane). The I-Au144 structure is viewed along a C3 axis; the D5-Au130 structure is viewed along a $\mathrm{C} 2$ axis. This comparison is based on the structures reported in Ref. 38 for the icosahedral $(144,60)$ cluster, and in Ref. 44 for the decahedral $(130,50)$ one. 